\title{
Collaborative Concept Mapping: Connecting with Research Team Capacities
}

\author{
Linda De George-Walker' ${ }^{1}$ and Mark A. Tyler ${ }^{2}$ \\ ${ }^{1}$ School of Human, Health and Social Sciences, Central Queensland University, Locked Bag 3333, Bundaberg, QLD 4670, Australia \\ ${ }^{2}$ School of Education and Professional Studies, Griffith University, Mt Gravatt Campus, 176 Messines Ridge Road, \\ Mt Gravatt Brisbane, QLD 4122, Australia
}

Correspondence should be addressed to Mark A. Tyler; m.tyler@griffith.edu.au

Received 19 November 2013; Accepted 30 March 2014; Published 1 June 2014

Academic Editor: Bernhard Schmidt-Hertha

Copyright (c) 2014 L. De George-Walker and M. A. Tyler. This is an open access article distributed under the Creative Commons Attribution License, which permits unrestricted use, distribution, and reproduction in any medium, provided the original work is properly cited.

\begin{abstract}
Concept mapping has generally been used as a means to increase the depth and breadth of understanding within a particular knowledge domain or discipline. In this paper we trace the deployment of collaborative concept mapping by a research team in higher education and analyse its effectiveness using the crime metaphor of motive, means, and opportunity. This case study exemplifies two iterations of the research team's collaborative concept map and shows how the process of the construction of such maps enabled the opportunity for team dialogue and coconstruction that was focused, hands-on, and visual. The concept mapping process provided the team with a meaning-making mechanism through which to share understandings and explore the team's potential capacities.
\end{abstract}

\section{Introduction}

In an effort to address the quality and impact of research, higher education institutions have invested in research capacity building initiatives including those that leverage the expertise and relationships within research collaborations and teams. Yet, the various methods and tools appropriate for understanding and building research capacity within research teams remain underexplored. This paper reports on a case study of a collaborative concept mapping process (CCM) with an educational research team located at a regional Australian university. We argue that collaborative concept mapping is an exercise that embodies effective capacity building processes by enabling exploration, articulation, and negotiation of shared motives and opportunities for research team development.

We undertake this journey by first conceptualising capacity and the building of research capacity as it appears in the literature. We then move to CCM and argue how it is well suited as a capacity building process. Next we introduce the case study per se, summarise the research team's first experience with $\mathrm{CCM}$, and highlight and explore the refinements made with the team's second attempt. The paper culminates with an analysis of CCM as a capacity building process in relation to the means, motives, and opportunity framework of Britton as deployed by James and Wrigley [1].

1.1. Conceptualising Capacity Building. Broadly, capacity building stimulates desired development and change for individuals, organisations, and communities. Yet, capacity building has an intangibility that "makes it the stuff of myth or magic" [2, page 210]. First, capacity building as a concept has been described as a mysterious, elusive, confused, and misinterpreted, with numerous definitions present in the literature [1-4]. Further, capacity building is considered to be undertheorised [3]. Harrow [2], however, concluded that although "the concept appears theoretically homeless," it is not necessarily atheoretical having found "temporary accommodation in a variety of literature" (page 226), for example, capacity building features in the literature on community development $[4,5]$; international aid $[1,3]$; public management and social policy $[2,6]$; health policy and practice $[7,8]$; and educational research $[9,10]$. Accordingly, capacity 
building has been variously theorised, for example, by means of stewardship theory (e.g., [2]), social capital theories (e.g., [11]), developmental systems theories, such as Bronfenbrenner's bioecological model (e.g., [8]), and transformational leadership (e.g., [5]). Given the range of contexts and conceptualisations it is hardly surprising that a single encapsulating definition and theory of capacity building remains elusive. Rather than considering this as an undesired situation to be rectified, definitional and theoretical eclecticism can be argued as necessary for the application of capacity building across the varied contexts in which it has been deployed.

No matter which definition, theory and conceptual lens is used to frame capacity building; a critical question remains: what is effective capacity building? James and Wrigley [1], adopting the crime metaphor of Britton, identify that effective capacity building in aid-based contexts requires attention to motive, means, and opportunity.

Analysing CCM by using this framework does offer a sleuthing kind of subtext to the development of greater understanding in relation to the happenings within and as a result of the process. In this case we are focused on the three aspects surrounding the doing of CCM and its relationship to the individuals involved (their motives), possibilities for actions (opportunities), and its effectiveness as a method (means). Specifically, motive here refers to individual, organisational, and community desire and incentives for change, including the construction of shared stakeholders' understandings about the nature of capacity building $[1,12]$. Opportunity refers to translating the work of capacity building into action and the availability of the required resources and support to do so; and means refers to methods for capacity building. Based on their review of international aid work, James and Wrigley [1, page ii] note that effective capacity building methods are those that

(i) retain and develop ownership;

(ii) are people-centred and relational;

(iii) engage with peoples' values and emotions;

(iv) use experiential approaches where appropriate;

(v) explicitly adapt to context and culture.

Similarly, others have noted that capacity building methods are a means rather than an end [12] and are necessarily relational "nurturing relatively stable patterns of social relations" [13] to empower "the potential of people to act together for a common community good" [4, page 38]. Effective capacity building methods are thus heterogeneous, process-oriented, and sociocultural practices that privilege and leverage relationships.

1.2. Building Research Capacity. In response to increased pressures in recent decades to improve research quality and impact, the international higher education sector has sought to build research capacity with various internal and cross-institutional initiatives implemented in the UK, USA, Australia, New Zealand, Scandinavia, and West Africa [15]. These initiatives have included institutional research policy development and investment in research infrastructure.
Other initiatives recognise that in addition to administrative and resourcing enablers for research activity, building research capacity requires building researcher capacity. One response to this has been researcher development programs that include technical skills training in areas such as research design and methods and proposal and grant writing [16]. Sterland [17], however, cautions that capacity building approaches that are reliant on skills development risk being too generic and may be unable to take account of specific context, stages and needs; may focus on immediate outcomes at the expense of longer term processes; and foster a simplified view of capacity building as components or parts disconnected from a whole organisation or context.

Some institutional research capacity building responses have consequently incorporated tailored research skills development activities within collaborative research networks and teams $[15,18-20]$. For example, in the developmental model of Wary and Wallace [21], researcher skills are built via research-related activities occurring within a mentoring model. While there is clearly value in peer and team processes for researcher development, there are also numerous challenges for collaborative and team research contexts, including failure to develop shared understandings about the motives, goals, frameworks, and ways of operating with regard to governance, communication, and conflict management $[16,20$, 22]. While the research capacity building literature identifies the nature of the problems to be addressed within teams and collaborations and highlights that solutions are ideally tailored and leverage social and relational practices in settings such as meetings and retreats, explicit methods and tools for fostering and understanding research capacity within teams and collaborations remain underexplored $[23,24]$.

In this paper, we will illuminate and add more substance to the myth and magic of capacity building as it relates to academic research team development and will argue that collaborative concept mapping, a process of concept mapping with small groups typically used for educational purposes $[25,26]$, is a valuable capacity building process for research collaborations. After reviewing what collaborative concept mapping is and the pertinent literature, we will present a case study on the use of collaborative concept mapping with an education research team. The aim of this case study research was (a) to describe the process and outcomes of the collaborative concept mapping method as the team members attempted to make explicit their theorising about team processes related to the direction and success of the team and (b) to analyse the capacity building potential of collaborative concept mapping in research contexts using the aforementioned motives, means, and opportunities framework as described by James and Wrigley [1] using Britton's conceptualisation.

\section{Collaborative Concept Mapping}

Concept mapping is the process of creating concept maps or diagrams that organise, represent, and create knowledge. Concept mapping can be utilised by individuals or small groups, the latter being typically referred to as collaborative 
concept mapping (CCM) [27]. The stages of CCM proceed in the same manner as individual concept mapping, as described by Novak and Cañas [26]. First, a clear context and knowledge domain is established by posing a focus question that is to be addressed during the concept mapping. Then around 20 key concepts are generated by participants in response to the focus question and are placed in a central "parking lot." An initial concept map is created from the parking lot concepts (although not all concepts must be used), arranging them in an hierarchal order from the most general to the most specific concepts and creating cross-links with linking words to form propositions. "Propositions are statements about some object or event in the universe, either naturally occurring or constructed. Propositions contain two or more concepts connected using linking words or phrases to form a meaningful statement" [26, page 1]. The process is a dynamic one, with the position of concepts and the cross-links able to be rearranged as necessary, and the map is always open to revision and "is never finished" [26, page 12].

Novak and Gowin [27] undertook seminal work on concept mapping demonstrating that concept mapping strategies enabled students to control the outcomes of certain education engagements. Through the process of sharing meaning with the teacher, and making concepts and connections explicit, students were shown to develop clearer pathways to the knowledge they were trying to capture. Watt [28], Chittenden [29], and Kuhn and Davidson [30] all reported instances of using this technique to increase learning toward deeper understanding. Novak and Cañas [26] suggest that concept mapping operates as a form of discovery learning.

Basque and Lavoie [25] reviewed 39 studies dating from the late 1980s that specifically investigated CCM. They cited evidence that CCM when compared with individual concept mapping produced better quality concept maps and benefited learning (e.g., [31, 32]). Further, they reported on studies showing that interactions observed during CCM were consistent with features of scientific discourse and included elaborated exchanges, coconstruction of meaning, and formation of alliances (e.g., [33,34]). Similarly, van Boxtel et al. [35] contend that the integrated conceptual framework or map that develops as part of the process of CCM provokes and supports discourse and elaboration, while enabling selfdetermined meaning making and personal reflection on an individual's (mis)understandings. Webb et al. [36] noted that the conditions of CCM that enable productive learning include opportunities for the clarification of understanding, content relevance, timeliness, and sufficient elaborations for the correction of misconceptions. More recently, Zwaal and Otting [37] found that groups who used concept mapping in the process of problem based learning "were more satisfied with the execution of...the decision-making process, and the communication within the group" (page 104), although in their study there was no conclusive evidence of increasing the group's ability to generate learning goals in relation to problems. Altogether, CCM studies have indicated that the process extends concept mapping processes and outcomes from individual learning to socially constructed meaning making.

\section{Case Study}

3.1. Background. The Capacity Building Research Network (CBRNetwork) is a multidisciplinary educational research team located in Australian regional university. The research experience of the 17 members of the CBRNetwork varies and includes research higher degree students, early- and midcareer researchers, and senior researchers working in a broad range of research interest areas pertaining to education and learning. Across this diversity of researcher interests and expertise exists a shared team focus on "capacity-building for emerging global, national and regional/community futures" which "encompasses sustainable and innovative practices, learning and development, lifelong learning and the development of self-determined communities" ("CBRNetwork", n.d.).

Five months after the formation of the research team in 2009, we facilitated an initial CCM exercise with the original smaller team of six researchers [14]. The purpose of the CCM activity was to assist the team to explore and document the meaning and operation of teamwork within the newly formed team. Accordingly, team members responded to the focus question of "How do we team?" and several complete propositions were evident in the partially completed concept map that emerged from the process (Figure 1), for example, (the concepts are in italics and linking words are nonitalicised):

(i) values are forgiveness, recognition, responsibility, reciprocity, trust, and respect;

(ii) authenticity, a requirement to establish and extend trust;

(iii) flexibility is lifelong learning is learning about new viewpoints and values is learning from others [14, page 65].

There were also some unexplored or only partially completed propositions, concepts, and links, most notably build capital and capacity and shared responsibility.

Towards the end of 2011, the research team successfully applied for formal status as a Faculty Research Centre, and as a consequence the membership of the team grew from seven to 17 members. Around this time, members expressed interest in revisiting the CCM exercise with the expanded team. Before proceeding with another CCM process, a formal and formative evaluation of the previous CCM process was conducted using an anonymous 10question online survey as documented in de George-Walker and Tyler [9]. The questions focused on member views about the value, challenges, and limitations of CCM for exploring concepts around teaming and the degree to which the propositions that emerged from the previous process reflected the current values, goals, and practices of the team. The ten team members who responded to the survey were positive about the CCM process and the overall position emerging from the survey was that CCM was a dynamic dialogical and visual tool that enabled individual reflection, collective coconstruction, and documentation of notions of team identity and teaming practices and facilitated a positive team climate. Also pertinent was the position that some 


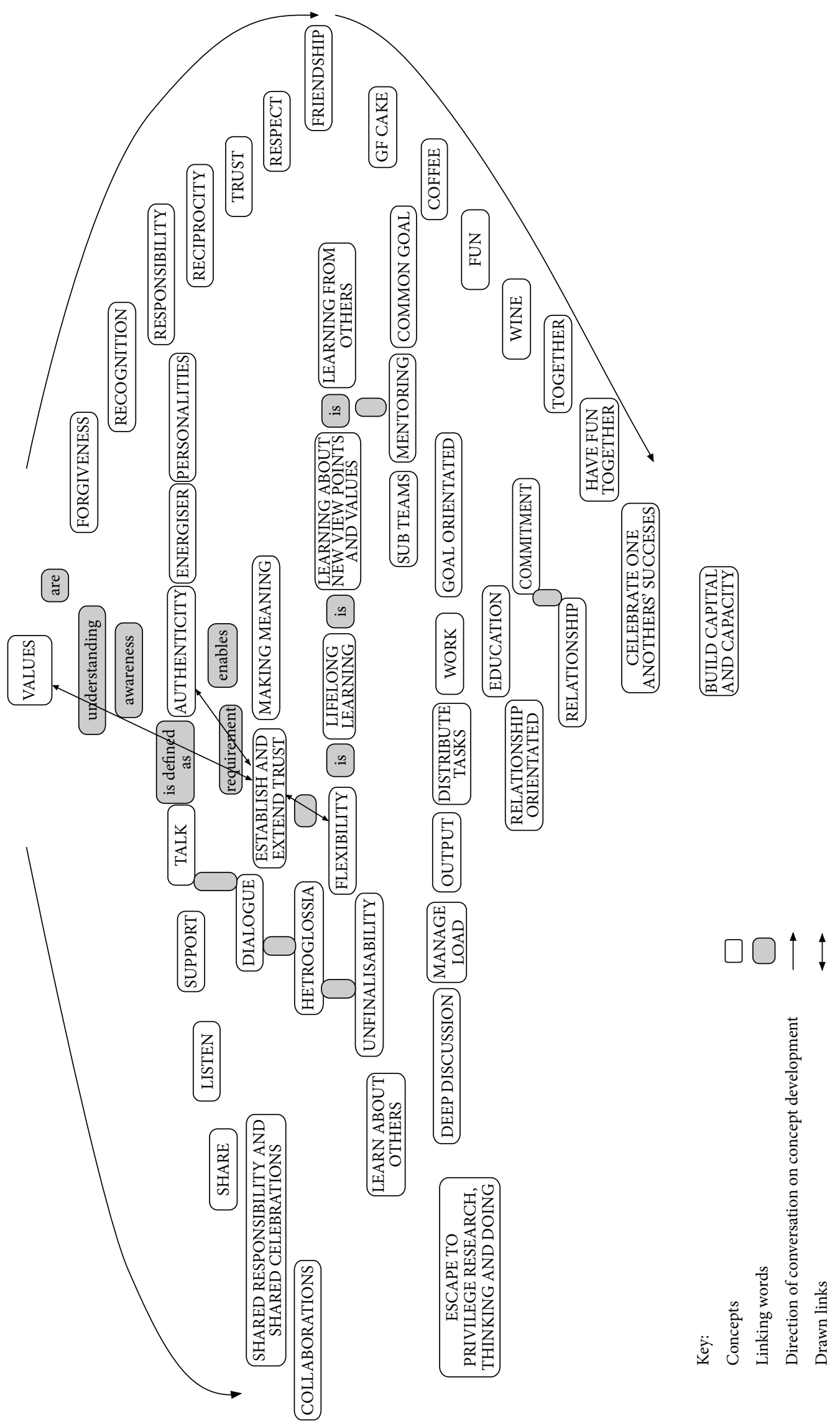

FIGURE 1: The first collaborative concept map produced by the research team. From "Collaborative concept mapping: an education research team leveraging their collaborative efforts" [14, page 66]. Reproduced and adapted with permission of publisher. 
additional needs of the team were not addressed in the initial CCM process; for example, there was limited exploration of the concepts of capacity, capacity building (as featured in the team name), leadership, and creativity and practical team processes such as communication and mechanisms for achievement of research goals. All survey respondents, including original and newer team members, indicated that it would be very useful to use CCM again with the expanded team membership to document and build shared notions around teamwork and to explore the other identified issues. Hence, a second culminating CCM exercise was conducted with the CBRNetwork in 2012 and is documented in the next sections.

3.2. CCM Process and Outcomes. The second CCM exercise was again facilitated by the authors of this paper, who at that time had become adjunct members of the team after taking up positions at other universities. Eight members of the team, not including the authors, participated in the process with a range of research experience from novice to expert researchers. Five participants were newer members and had not participated in the first CCM process. The CCM process occurred onsite at the regional campus where the majority of team members were located. The process occurred over a three-hour period with a 30-minute break around midway through the process.

The process began with the facilitators providing an overview of concept maps and the process of CCM and summarising the first CCM process and outcomes as overviewed in the previous section. Acknowledged by the group was that in the previous map there was a lack of prepositions and verbs that left certain agreed upon concepts unfinalised as propositions. For example, in Figure 1, the concepts talk, dialogue, hetroglossia, and unfinalisability are linked, but this link remains unspecified. Hence, these concepts do not make a knowledge proposition. The group was encouraged to focus in this CCM exercise on the building of complete propositions. The hierarchal ilk of the exercise was also reiterated noting that the top concept was to be shaped from the question, which was again initially concerned with notions of teamwork but was extended with the agreement of the group to include a focus on research outcomes upon which team funding was contingent: "How do we team in order to meet CBRNetwork's key performance indicators?"

The CCM process initially proceeded in a typical manner as outlined earlier in this paper. A parking lot was established and individuals contributed concepts by talking in various sized groups, comparing ideas and reviewing. Approximately 42 concepts were generated in 10 minutes. The facilitators then requested the group review a large number of concepts, and if appropriate, to group them under similar themes and label them with an overarching concept. This invited the group to observe any repetition in concepts and what this might mean with regard to the importance of various concepts and also to begin considering the hierarchical and cross-linked nature of the generated concepts. The broader concepts that emerged during this theming stage were management, mentoring, networking, academic writing and publishing, personal growth, capacity building, constraints, profile, lateral thinking, and outcomes and outputs. Taking the example of management, some of the concepts identified by the group as subordinate concepts were shared leadership, strategic planning, long-term goals, prioritising, setting limits, identifying opportunities, cost-benefit analysis, project management, budget, administrative support, corporate support, and sharing resources.

Next, participants were invited to begin assembling the concept map on the whiteboard using the sticky notes and whiteboard markers to record the cross-links and linking words. A team member volunteered as the scribe and began moving the concepts around on a large whiteboard whilst others looked on and engaged in general discussion about these concepts and suggested changes. The team initially chose the concept of teaming as one of the top concepts but quickly moved on to discuss notions of leadership and how it might be distinguished from governance. One complex proposition began to arise as the team attempted to link the outcomes from the discussions of teaming and leadership in a concept map:

team develops strategic direction that guides governance, which is done by management committee that reports to Faculty/University policy which impacts management committee.

Another branch of the map formed by the participants offered an incomplete proposition: team needs motivation. Motivation was then connected to strategic direction but without a joining preposition. Motivation was identified by one team member as nonconcrete and argued what was really sought by the team was the driver or machinery of motivation for research. In brief discussion about the "what" of research, the different paradigms of research were acknowledged and it was identified that for this team, research produced change and informed practice. The suggestion made was that the scholarship of capacity building might be the motivational driver for the team's practice-oriented research.

The unfolding concept map was then abandoned by the group and a question was written on the board "What is capacity building?" After some discussion it was agreed by the group that the scholarship of capacity building should be the central component to the somewhat slippery notion imbedded in the group's doing-which is also the primary nomenclature in the group's name. With this epiphany, scholarship of capacity building ( $S$ of $C B$ ) was chosen as a central component of a new map that departed from the typical concept map form and took the form of a circle with other concepts circling the central position of $S$ of $C B$. The concepts of profile, $P h D$, writing, and external funds were interconnected with prepositions to make a seemingly meaningful set of propositions and the concept map began to look more like a conceptual model. Nevertheless, useful propositions emerged; for example, the $S$ of $C B$ attracts $P h D$ (students); the $S$ of $C B$ develops profile that enhances and attracts external funds.

The scribe then reconstituted this "map" into a set of concentric circles with $S B$ of $C$ at the center. The concept of relationships was closest to the center, and external funds, 
PhDs, and outputs (writing and publishing) in the next circle, with profile being the furthest, were removed from the center. No propositions were built within this figure. This figure was then again reconceptualised by the participants into a threedimensional cone shape with the "pointy end" housing the central concept of the $S$ of $C B$ (Figure 2). The cone figure was placed horizontally to fit the confinements of the white board, but its positioning was intended as vertical, reflecting the hierarchal tenant of concept mapping. At the base of the cone was profile. The skin of the cone carried the verbs produces and enhances, seemingly producing the proposition: $S$ of $C B$ produces and enhances profile. Other concepts-outputs, PhDs, and external funds-were positioned centrally on the cone and just below the "skin" in between $S$ of $C B$ and profile. These were linked to other concepts, still written on sticky notes and linked by the phrase "are developed by." These links produced the proposition that outputs, PhDs, and external funds are developed by mentoring, coauthoring, engagement, professional conversations, according to her/his activity, cross-disciplinary connections, support, and flexibility. A central core to the cone was prominent running from top to bottom with the concept of relationships written on it. The core too had a thin skin labelled as governance. The core or relationships was connected by an arrow to what appeared as an important proposition for the team: $S$ of $C B$ is more productive when relational. It is noted that this figure may be interpreted as a model. Nevertheless, we continue to use the nomenclature of "concept map." This is because the figure was named as a concept map by the team and second it displays many of the characteristics of a concept map; for example, it is hierarchal and knowledge propositions are clearly evident.

3.3. Analysis of CCM as a Capacity Building Process. The data used for the analysisof CCM as a capacity building process included the completed concept map (Figure 2), photographs of the concepts, and maps that were created on the sticky notes and whiteboard as the process unfolded, an audio recording of the session, a note form transcription of the audio recording that assisted in navigating the audio recording during the process of the analysis, and the authors' own observations during the session. A thematic content analysis of the data was conducted according to the means, motives, and opportunity framework of Britton that James and Wrigley [1] had deployed. The researchers were, however, also open to unexpected themes that might be evident in the data.

3.3.1. Motive and Opportunities. James and Wrigley [1] argue that for effective aid-based capacity building, there must be an adequate internal motive for change and development, and this necessarily involves exploration, articulation, and negotiation of a clear and consensual "prize" among stakeholders. They note the importance of leadership for development and change, but shared motivation and purpose is an essential element for effective capacity building and is "assisted when people feel they have had the opportunity to contribute meaningfully to the identification of both the need to change and the appropriate ways forward" (page 10). Developing shared purpose and motivation involves unearthing and addressing individual and shared values, beliefs, agendas, and interests; matters that can often be complex, emotion-laden, and challenging to discuss, let alone reaching consensus on. We argue that to successfully build the capacities of research teams there must be a shared motive and that CCM is a valuable tool that enables exploration, articulation, and negotiation of purpose.

First, with regard to shared purpose for this team, it was clear from their participation in and evaluation of the first CCM process that the team had a strong shared motive to understand team processes as they applied to their future direction as a research team and to revisit the topic with the expanded membership to allow voices and views of newer members to be heard. The team also had a shared commitment to capacity building research as evidenced in the team name and shared goals in the form of key performance indicators around publication outputs, grant applications, and successes and attracting research higher degree students. What was less clear for the team was a shared conceptual framework guiding their approach to team research activities. This was a concern raised regularly during team meetings and in the evaluation of the first CCM process and had been identified by the team as weakness they needed to resolve in order to fully function and to successfully achieve their research goals. The issue was such that it prompted the team to commission a discussion paper about the various conceptualisations of capacity building and a possible direction for the team. The way forward on this issue had, however, continued to elude the team and the lack of a clear conceptualisation of capacity building was again raised during this CCM process as a barrier to the team achieving their goals and establishing their profile in the capacity building field.

As noted earlier in the description of this CCM process, there was a fundamental and shared philosophical shift evident as the team focused their efforts towards understanding the meaning of the scholarship of capacity building. Up until this point the team was at an impasse, recognising the need for shared resolution of this conceptual block, but struggling with how to move forward in a manner that would be inclusive of all team member's skills, knowledge, philosophies, and abilities. As a result of this CCM process the team agreed that the diversity of theoretical and conceptual frameworks the team members contributed was a strength in their approach to the understanding and the application of capacity building and that settling for one conceptualisation of capacity building was not only unnecessary, but also would potentially limit the team. Further, the team agreed that the relational nature of capacity building was fundamental to their scholarship of capacity building, which is clearly evident in the concept map (Figure 2) with the propositions that the $\mathrm{S}$ of $\mathrm{CB}$ produces and enhances relationships; the $\mathrm{S}$ of $\mathrm{CB}$ is relational and relationships are developed by mentoring, coauthoring, engagement, professional conversation, each according to his or her own activities, cross-disciplinary connections, support, and flexibility.

This team's development of shared understandings of the meaning of the scholarship of capacity building and the value 


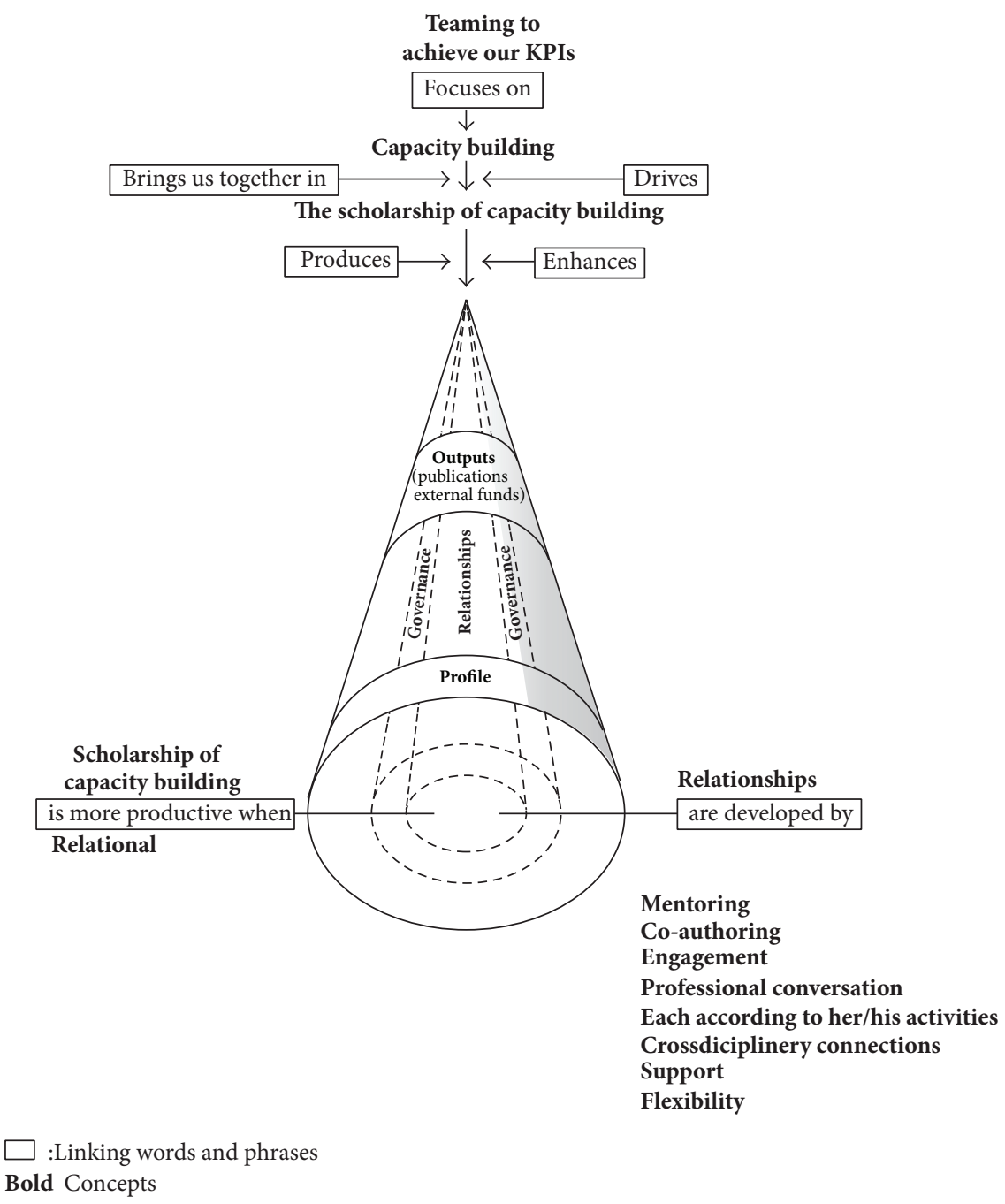

FIgURE 2: The second collaborative concept map produced by the research team.

of an eclectic conceptualisation of capacity building appeared to strengthen their sense of collective motive and purpose. The importance of a common bond, mindset, or framework has been identified in the literature as not only adding to the depth of scholarly work but also building team spirit, trust, and collegiality [38, 39]. Further, embracing of definitional, conceptual, and theoretical eclecticism in regard to capacity building appears necessary for this team to successfully apply and research capacity building across the varied contexts with which it engages [40].

James and Wrigley [1] in their discussion of motives for capacity building caution against externally driven development note that there will be little incentive for stakeholders to be involved in this situation. This research team was cognisant of the external systemic drivers for the achievement of research outcomes, namely, ongoing Faculty funding being contingent upon achieving the key performance indicators. The Faculty role can be viewed as similar to the funding donor role in aid-based capacity building endeavours in that donors may view capacity building in terms of ambitious short-term goals and their owns needs for accountability in the form of proposals, reports, and financial management processes. Similar to the notion of "systemic disincentives" for capacity building that James and Wrigley identify in the aid-system, the team did refer to the characteristics of institutional administration and reporting requirements that may be barriers to the achievement of the research goals and noted that the time spent on these tasks can take away from the work of producing publications, applying for grants, and developing the partnerships often necessary to progress these outcomes. James and Wrigley contend that an appreciation of these restraints is important, so these can be directly addressed. During the CCM process, the discussion about the difference between governance and leadership assisted the team to identify the importance of the team's governance to "shield" and deflect certain Faculty and organisational requirements (such as financial reporting) from those at the "coal face," so they can get on with the business of achieving the key performance indicators. Here we can see the strength of the team's internal (versus external) motive 
for development towards achieving their goals and how they can acknowledge the importance of, but not succumb to, institutional pressures.

In the process of clarifying and negotiating a shared conceptualisation of the scholarship of capacity building, and also the differences between leadership and governance, the team was able to reach some consensus on previously troubling issues and identify the importance of harnessing the diversity of members and the team governance processes towards achieving team goals and establishing the team as a valuable presence in the field of capacity building. However, many capacity building efforts fail because of lack of time or resourcing for ongoing processes, legitimacy, or encouragement to implement change [1]. This team was well aware of these challenges and recognised similar challenges, namely, uncertainty of funding and time poor researchers, as risk factors for successful implementation.

3.3.2. Method. As described in the literature review, James and Wrigley [1] note that effective capacity building methods are those that retain and develop ownership, are peoplecentered and relational, engage with peoples' values and emotions, use experiential approaches where appropriate, and explicitly adapt to context and culture. We argue that the CCM process, through the opportunity for elaborated dialogue and coconstruction in the context of a focused, hands-on, and visual meaning making activity, embodies all of these characteristics and provided this research team with a mechanism through which the "prize" of a shared motive and understanding of the opportunities could be explored, articulated, negotiated, and also documented in the form of the concept map.

The CCM process thus has potential as a capacity building method or tool, yet as with all tools, the CCM facilitator, whether internal or external to the group, must be sufficiently skilled in the same ways that James and Wrigley [1] note that capacity building accomplices must be skilled. That is, they must be able to foster participant ownership of the process; encourage dialogue and reflection; manage emotions, tensions, and power dynamics; and be culturally and contextually sensitive [1]. James and Wrigley also refer to the importance of balancing structure and flexibility, an issue which was highly pertinent during this case study as the concept map did not take the shape typical of a concept map. Had we, as facilitators, insisted on the typical concept map format, one of the key tenets of effective capacity building approaches, that they retain and develop ownership for participants would have been lost. Sterland [17] observed that "it is critical that practitioners take the point of departure... [and] adapt their methods and facilitation during each capacity building process" (page 4). And in the end the shape of the map did not hinder the development of clear propositions that reflected the groups shared conceptualisation of capacity building and motives.

\section{Conclusion}

In this paper we have given an account of one academic research team's attempt to use CCM, typically an educational tool, to conceptualise and make explicit the "how" of developing their research capacity. The efficacy of the CCM process for capacity building was further scrutinised using the aidbased motive, means, and opportunity framework [1]. The case study nature of this inquiry means it is not possible to generalise the findings about the process and outcomes to other research teams. The richness of the findings has, however, allowed for an in-depth exploratory study of the novel application of CCM and supported the potential of CCM as a tool for building research team capacity.

The findings of this study indicated that features of the CCM method are characteristic of those identified as necessary for effective capacity building processes; that is, the process is an experiential one that develops ownership, is people-centred and relational, engages with peoples' values and emotions, and can adapt to context and culture. CCM in this case study enabled shared exploration, articulation, and negotiation of intentions and opportunities for the research team and produced a collective concept map that conceptualised and documented this-a map that is a bold artefact of purpose whose focused intent yet offers a shared construct for critical reflection. What the map highlighted was a clear motive and purpose by team members to understand their team and commit to shared research goals, such as increasing publication outputs and attracting research higher degree students, but the map also documented a shared commitment by the team members to the capacity building of the individuals, groups, and communities with which they research and to the scholarship of capacity building. In terms of opportunity, using the CCM process enabled, among other things, an accepting appreciation of possible constraints and the opportunities to deal openly with troubling concerns, for example, the tensions that arose between leadership and governance. The use of CCM to leverage the expertise and relationships within research teams for building research capacity is highly significant, particularly when we consider how it focused effort and intent into a collaborative, reasoned, and balanced response to the research imperative.

\section{Conflict of Interests}

The authors declare that there is no conflict of interests regarding the publication of this paper.

\section{Acknowledgment}

The authors wish to acknowledge the members of the Capacity Building Research Network (CBRNetwork) who participated in the collaborative concept mapping process reported in this paper.

\section{References}

[1] R. James and R. Wrigley, "Investigating the mystery of capacity building: learning from the Praxis programme," Praxis Paper 18, International NGO Training and Research Centre (INTRAC), Oxford, UK, 2007, http://www.intrac.org/data/ files/resources/415/Praxis-Paper-18-Investigating-the-Mysteryof-Capacity-Building.pdf. 
[2] J. Harrow, "Capacity building” as a public management goal: myth, magic or the main chance?" Public Management Review, vol. 3, no. 2, pp. 209-230, 2010.

[3] S. Kenny and M. Clarke, Challenging Capacity Building: Comparative Perspectives Rethinking International Development, Palgrave Macmillan, New York, NY, USA, 2010.

[4] B. Kwan, J. Frankish, D. Quantz, and J. Flores, A Synthesis Paper on the Conceptualization and Measurement of Community Capacity, Institute, of Health Promotion Research, University of British Columbia, Vancouver, Canada, 2003, http://www.ufv.ca/Assets/BC+Centres+(CRIM)/Non-Profit+ Development/Articles/Best+Practises+Community+Capacity +Building/A+Synthesis+Paper.pdf.

[5] J. McCalman, K. Tsey, R. Kitau, and S. McGinty, “Bringing us back to our origin": adapting and transferring an Indigenous Australian values-based leadership capacity-building course for community development in Papua New Guinea," Community Development, vol. 43, no. 3, pp. 393-408, 2012.

[6] E. Kendall, H. Muenchberger, N. Sunderland, M. Harris, and D. Cowan, "Collaborative capacity building in complex community-based health partnerships: a model for translating knowledge into action," Journal of Public Health Management and Practice, vol. 18, no. 5, pp. E1-E13, 2012.

[7] B. R. Crisp, H. Swerissen, and S. J. Duckett, "Four approaches to capacity building in health: consequences for measurement and accountability, Health Promotion International, vol. 15, no. 2, pp. 99-107, 2000.

[8] K. B. Mistry, C. S. Minkovitz, A. W. Riley et al., "A new framework for childhood health promotion: the role of policies and programs in building capacity and foundations of early childhood health," American Journal of Public Health, vol. 102, no. 9, pp. 1688-1696, 2012.

[9] L. de George-Walker and M. A. Tyler, "Building capacity for collaborative research: revisiting a collaborative concept mapping exercise within the context of a research team," in Constructing Capacities: Building Capabilities Through Learning and Engagement, P. A. Danaher, L. de George-Walker, R. Henderson et al., Eds., pp. 239-255, Cambridge Scholars, Newcastle upon Tyne, UK, 2012.

[10] C. Mitchell and L. Sackney, Profound Improvement: Building Capacity for a Learning Community, Routledge, Abingdon, UK, 2011.

[11] K. Malik and S. Wagle, "Civic engagement and development: introducing the issues," in Capacity for Development: New Solutions to Old Problems, S. Fukuda-Parr, C. Lopes, and K. Malik, Eds., pp. 85-100, Earthscan, London, UK, 2002.

[12] H. A. Mengers, "Making urban sector lending work; lessons from a capacity building programme in Karnataka, India," Habitat International, vol. 24, no. 4, pp. 375-390, 2000.

[13] J. F. Ohiorhenuan and S. M. Wunker, Capacity Building Requirements for Global Environmental Protection, Global Environment Facility, Washington, DC, USA, 1995.

[14] M. A. Tyler and L. de George-Walker, "Collaborative concept mapping: an education research team leveraging their collaborative efforts," in SustaInIng Synergies: Collaborative Research and ResearchIng Collaboration, C. H. Arden, P. A. Danaher, L. de George-Walker et al., Eds., pp. 54-71, Post Pressed, Mount Gravatt, Qld, Australia, 2010.

[15] J. Murray and A. Pollard, "International perspectives on research capacity building," British Journal of Educational Studies, vol. 59, no. 3, pp. 219-224, 2011.
[16] A. M. Barrett, M. Crossley, and H. A. Dachi, "International collaboration and research capacity building: learning from the EdQual experience," Comparative Education, vol. 47, no. 1, pp. 25-43, 2011.

[17] B. Sterland, "Metaphor and analogy: creating meaning and understanding complexity," Praxis Note 9, International NGO Training and Research Centre (INTRAC), Oxford, UK, 2005, http://www.intrac.org/data/files/resources/100/Praxis-Note-9Metaphor-and-Analogy.pdf.

[18] R. Leitch, "Harnessing the slipstream: building educational research capacity in Northern Ireland," Journal of Education for Teaching, vol. 35, no. 4, pp. 355-371, 2009.

[19] B. Ridley, "Educational research culture and capacity building: the case of Addis Ababa University," British Journal of Educational Studies, vol. 59, no. 3, pp. 285-302, 2011.

[20] S. Scott, “Team working: an anathema to academics?" in In Practice, vol. 1, pp. 1-4, 2009, http://www.lfhe.ac.uk/publications/ inpractice21.pdf.

[21] A. Wary and M. Wallace, "Accelerating the development of expertise: a step-change in social science research capacity building," British Journal of Educational Studies, vol. 59, no. 3, pp. 241-264, 2011.

[22] G. Castillo, Research Partnerships: Issues, Lessons, Results and Dreams for Sustainable Development, ODI Agricultural Research and Extension Network, London, UK, 1997.

[23] T. M. Paulus, M. Woodside, and M. F. Ziegler, "I tell you, it's a journey, isn't it?" understanding collaborative meaning making in qualitative research," Qualitative Inquiry, vol. 16, no. 10, pp. 852-862, 2010.

[24] S. M. Ritchie and D. L. Rigano, "Solidarity through collaborative research," International Journal of Qualitative Studies in Education, vol. 20, no. 2, pp. 129-150, 2007.

[25] J. Basque and M. Lavoie, "Collaborative concept mapping in education: major research trends," in Proceedings of the 2nd International Conference on Concept Mapping, A. J. Cañas and J. D. Novak, Eds., San José, Costa Rica, 2006, http://cmc.ihmc.us/cmc2006Papers/cmc2006-p192.pdf.

[26] J. D. Novak and A. J. Cañas, "The theory underlying concept maps and how to construct and use them (revised ed.)," Tech. Rep. IHMC CmapTools 2006-01, Florida Institute for Human and Machine Cognition, http://cmap.ihmc.us/Publications/ ResearchPapers/TheoryUnderlyingConceptMaps.pdf.

[27] J. D. Novak and D. B. Gowin, Learning How to Learn, Cambridge University Press, Cambridge, UK, 1984.

[28] J. Watt, "Organising information: concept maps and data charts," Classroom, vol. 22, no. 6, pp. 26-27, 2002.

[29] E. Chittenden, "Building conceptual bridges," Teacher, vol. 178, pp. 58-60, 2007.

[30] S. Kuhn and J. Davidson, "Thinking with things, teaching with things," Qualitative Research Journal, vol. 7, no. 2, pp. 63-75, 2007.

[31] P. A. Okebukola and O. J. Jegede, "Cognitive preference and learning mode as determinants of meaningful learning through concept mapping," Science Education, vol. 72, no. 4, pp. 489500, 1988.

[32] N. Stoyanova and P. Kommers, "Concept mapping as a medium of shared cognition in computer-supported collaborative problem solving," Journal of Interactive Learning Research, vol. 13, no. 1, pp. 111-133, 2002.

[33] W. M. Roth and A. Roychoudhury, "The social construction of scientific concepts or the concept map as conscription device 
and tool for social thinking in high school science," Science Education, vol. 76, no. 5, pp. 531-557, 1992.

[34] S. Sizmur and J. Osborne, "Learning processes and collaborative concept mapping," International Journal of Science Education, vol. 19, no. 10, pp. 1117-1135, 1997.

[35] C. van Boxtel, J. van der Linden, E. Roelofs, and G. Erkens, "Collaborative concept mapping: provoking and supporting meaningful discourse," Theory into Practice, vol. 41, no. 1, pp. 40-46, 2002.

[36] N. M. Webb, S. H. Farivar, and A. M. Mastergeorge, "Productive helping in cooperative groups," Theory into Practice, vol. 41, no. 1, pp. 13-20, 2002.

[37] W. Zwaal and H. Otting, "The impact of concept mapping on the process of problem-based learning," Interdisciplinary Journal of Problem-Based Learning, vol. 6, no. 1, pp. 104-128, 2012.

[38] A. M. Liggett, C. E. Glesne, A. P. Johnston, S. B. Hasazi, and R. A. Schattman, "Teaming in qualitative research: lessons learned," Qualitative Studies in Education, vol. 7, no. 1, pp. 77-88, 1994.

[39] P. Woods, M. Boyle, B. Jeffrey, and G. Troman, "A research team in ethnography," International Journal of Qualitative Studies in Education, vol. 13, no. 1, pp. 85-98, 2000.

[40] D. Tedmanson, "Whose capacity needs building? Open hearts and empty hands: reflections on capacity building in remote communities," in Proceedings of the International Critical Management Studies Conference, Postcolonialism Stream, University of Cambridge, 2005, http://www.mngt.waikato.ac. nz/ejrot/cmsconference/2005/abstracts/postcolonialism/Tedmanson.pdf.

[41] CBRNetwork, http://www.usq.edu.au/education/research/current-research/cbrnetwork. 

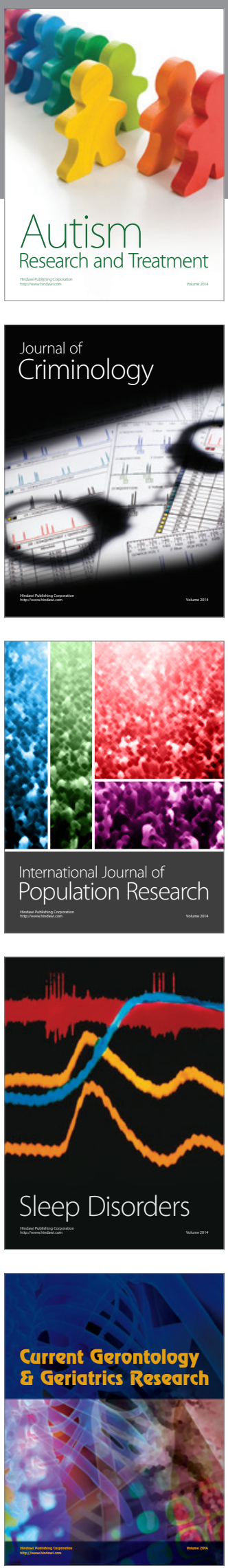
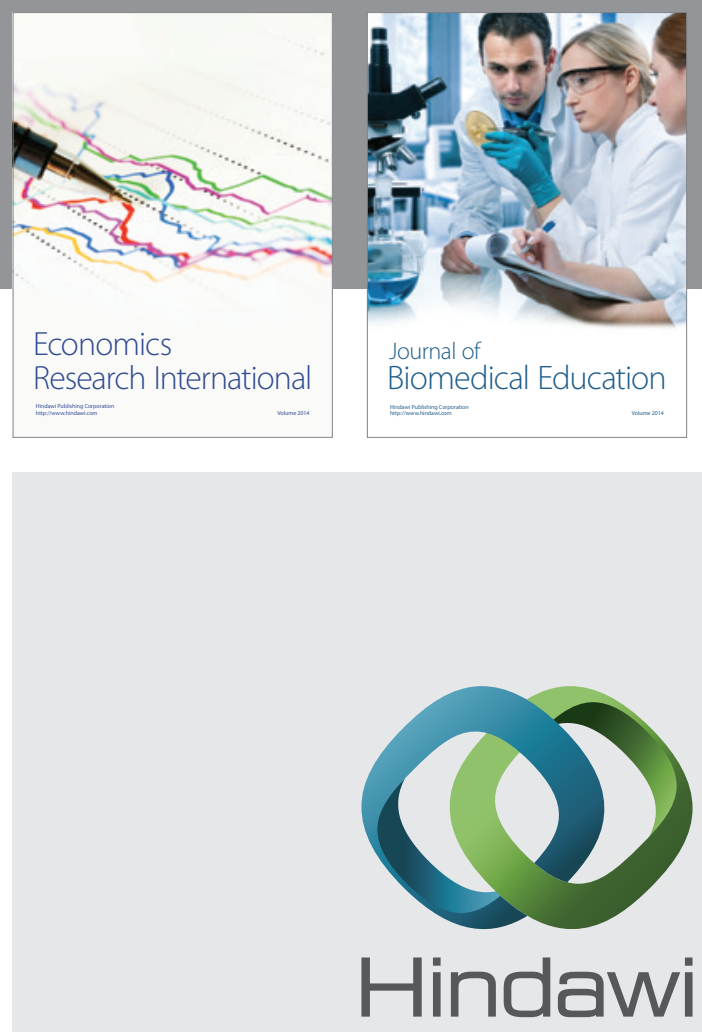

Submit your manuscripts at

http://www.hindawi.com
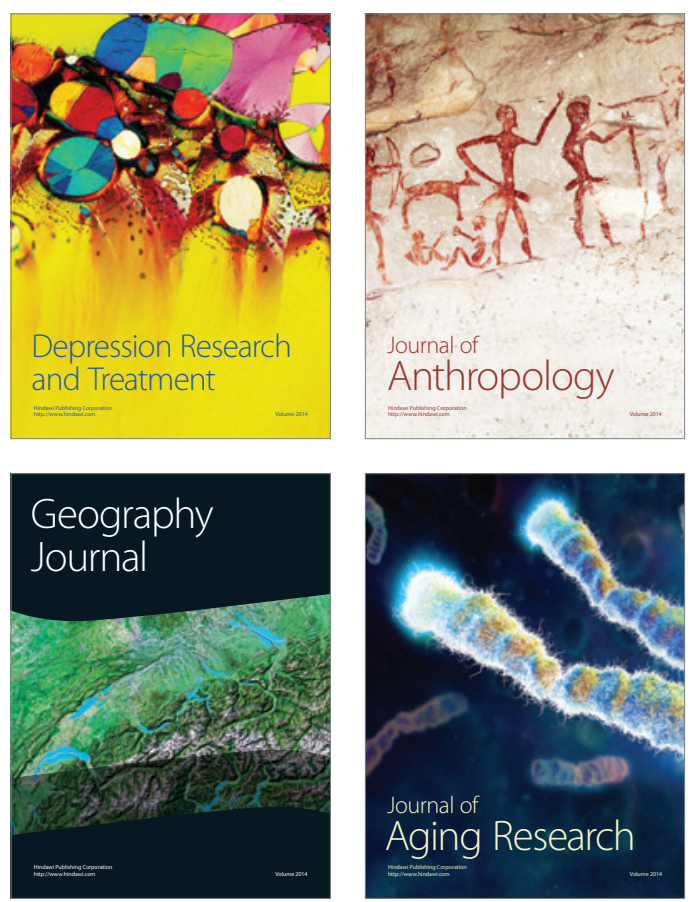
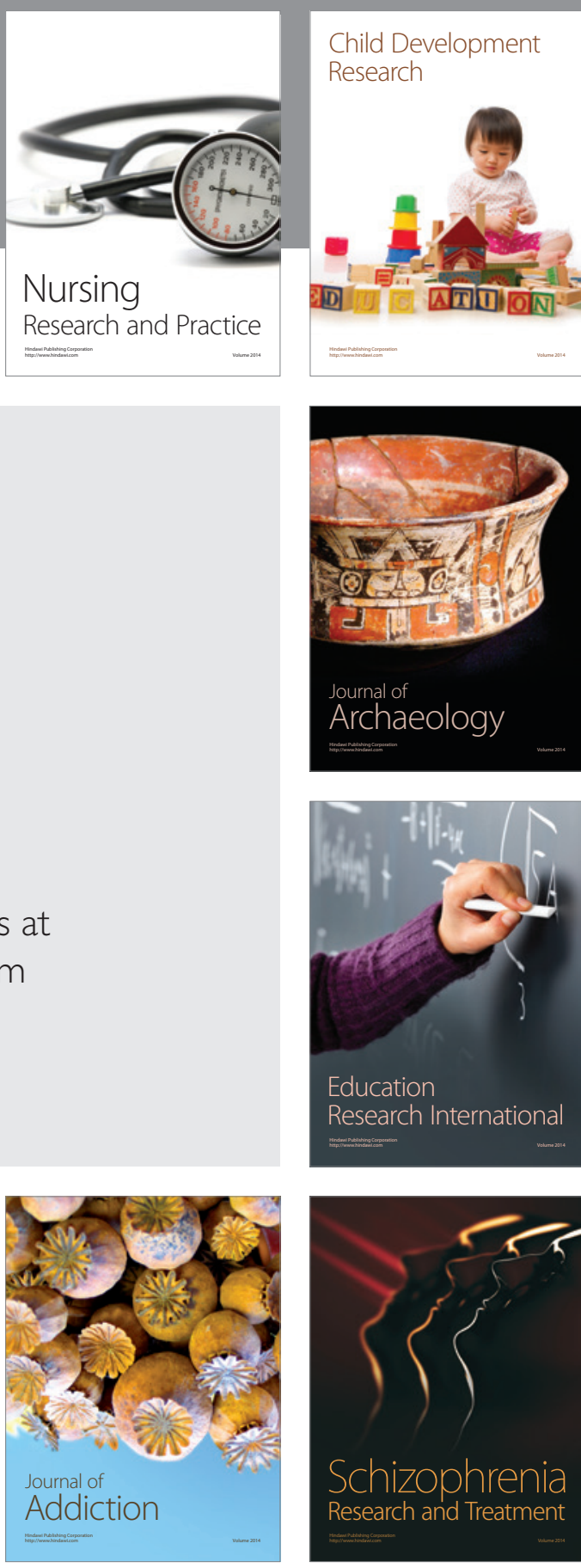

(D)
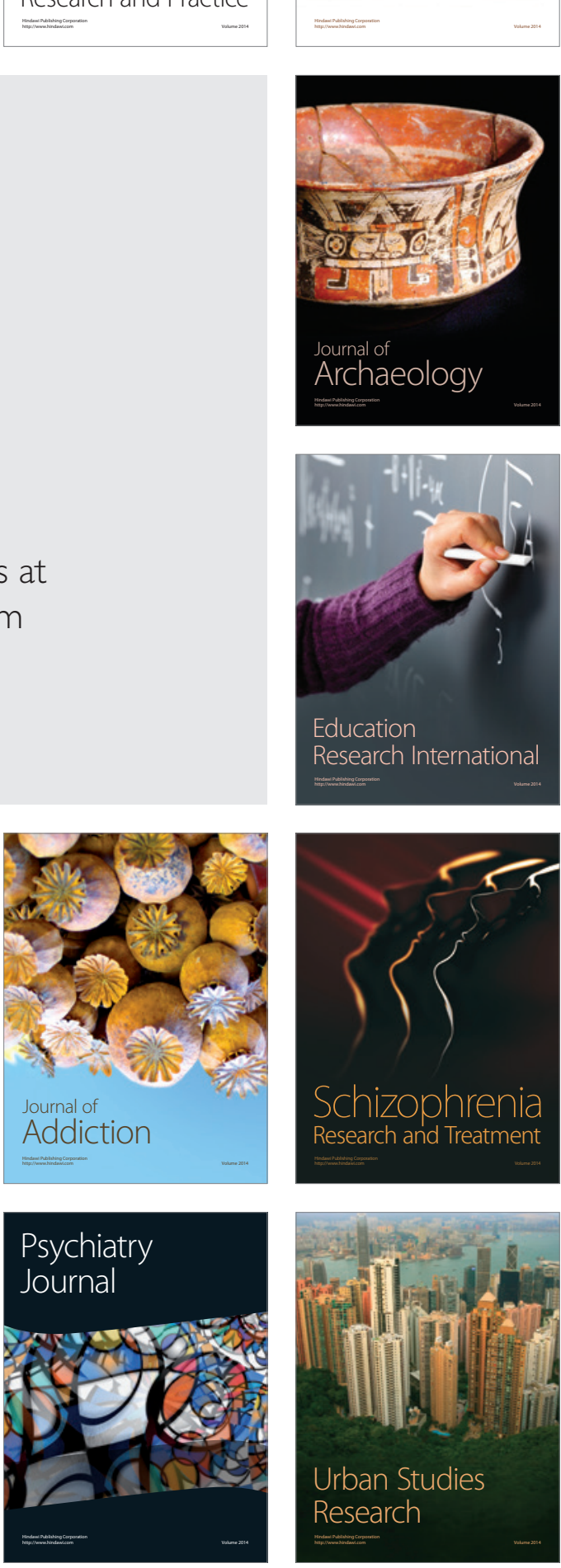